\title{
Guanosine supplementation reduces apoptosis and protects renal function in the setting of ischemic injury
}

\author{
K.J. Kelly, Zoya Plotkin, and Pierre C. Dagher \\ Department of Medicine, Division of Nephrology, Indiana University, Indianapolis, Indiana, USA \\ Address correspondence to: Pierre Dagher, Department of Medicine, Division of Nephrology, 1120 South Drive, FH 115, \\ Indianapolis, Indiana 46202, USA. Phone: (317) 278-2867; Fax: (317) 274 8575; E-mail: pdaghe2@iupui.edu.
}

Received for publication April 12, 2001, and accepted in revised form September 11, 2001.

Ischemic injury to the kidney is characterized in part by nucleotide depletion and tubular cell death in the form of necrosis or apoptosis. Recently, we linked anoxia-induced apoptosis in renal cell cultures specifically to the depletion of GTP. We therefore hypothesized that enhancing GTP repletion in vivo might protect function by reducing apoptosis in postischemic tubules. Male C57 black mice (the "I" group of animals) underwent bilateral renal artery clamp for 32 minutes to induce ischemia and then received either normal saline ("NS") or guanosine ("G"). After 1 hour of reperfusion, renal GTP levels in NS/I were reduced to nearly half of those in sham operated mice, whereas these levels were nearly unchanged in G/I mice. Morphologic examination of tubular injury revealed no significant differences between the two groups. However, there was a significant reduction in the number of apoptotic tubular cells in the medulla in the G/I group as compared with the NS/I group. At 24 hours, creatinine was significantly elevated in the NS/I group, compared to the G/I group. We conclude that guanosine protects against renal ischemic injury by replenishing GTP stores and preventing tubular apoptosis.

J. Clin. Invest. 108:1291-1298 (2001). DOI:10.1172/JCI200113018.

\section{Introduction}

Acute ischemic renal injury is a frequent clinical problem associated with high mortality (1). In recent years, there have been significant insights into the pathophysiology of renal ischemia $(2,3)$. Important vascular and hematologic factors involved include endothelins (4), nitric oxide (5), leukocytes (6), and growth factors (7). Nevertheless, there are still no accepted specific treatments for renal ischemia, and there has been little improvement in survival in the last 40 years (8).

Ischemic injury to tissues triggers a complex series of metabolic disturbances, often culminating in cell death. Furthermore, the importance of sublethal injury to tubular cells as it relates to the phenotype of cell death is increasingly appreciated (9). Many intracellular mediators have been implicated in altering the balance between apoptosis and necrosis. These include ATP depletion; cell $\mathrm{pH}$ and calcium; and phospholipid messengers such as ceramide, phospholipase A2, and reactive oxygen species. The relative importance of these mediators is tissue specific but their induction of apoptosis proceeds primarily via the intrinsic mitochondrial pathway.

Data showing the importance of nucleotide depletion in initiating cell death stems largely from cell culture studies. Using well-established models of mitochondrial poisons or anoxia, these studies have correlated the severity of ATP depletion with the phenotype of cell death (10). Mild to moderate ATP depletion triggers apoptosis while severe depletion results in necrosis. These studies fall well within the accepted paradigm of apoptosis having an absolute requirement for ATP. However, the impact of ischemiainduced changes in other nucleotides has not been extensively investigated (11).

Recently, we have documented remarkable changes in cellular GTP levels during chemical anoxia in vitro and renal ischemia in vivo (12). The decline in GTP levels paralleled ATP levels and was as severe. We further developed models of selective nucleotide depletion of graded severity and correlated the induction of apoptosis in LLC-PK1 renal epithelial cells solely with the depletion of GTP. ATP depletion of any severity, if unaccompanied by GTP depletion, resulted only in necrosis.

Here we extend our in vitro findings to evaluate the role of GTP in apoptosis during renal ischemia in vivo. Because we have documented previously profound depletion of GTP during renal artery clamp in the rat (12), we investigated whether enhancing GTP levels during reperfusion alters the induction of apoptosis in postischemic kidneys. Our results show that renal GTP levels are important determinants of the phenotype of cell death after renal ischemia. Furthermore, they underscore the critical role of apoptosis as a determinant of the functional injury to the kidneys after an ischemic insult.

\section{Methods}

Renal ischemia. All animal experimentation was conducted in conformity with the Guiding Principles for 


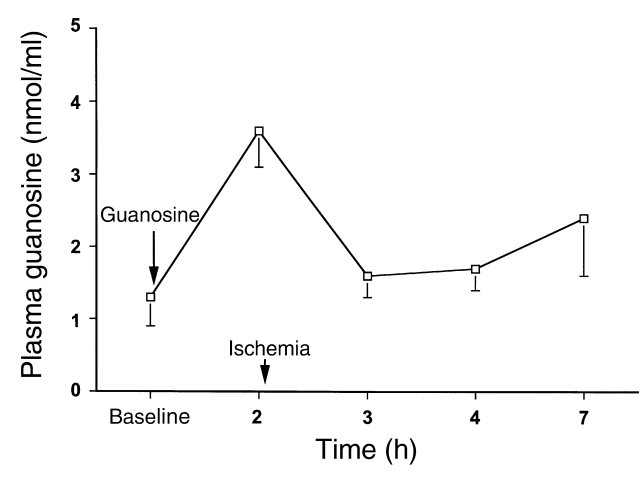

Figure 1

Effects of intraperitoneal guanosine on plasma guanosine levels. Animals received $30 \mathrm{mg} / \mathrm{kg}$ guanosine intraperitoneally 2 hours before bilateral renal ischemia/reperfusion. Blood was collected at various time points and plasma guanosine levels determined by HPLC as detailed in Methods. Values are means $\pm \operatorname{SE}(n=3)$.

Research Involving Animals and Human Beings. Guanosine (Sigma Chemical Co., St. Louis, Missouri, USA), $30 \mathrm{mg} / \mathrm{kg}$ in $0.9 \% \mathrm{NaCl}$, or an equal volume of $0.9 \% \mathrm{NaCl}$ was administered via intraperitoneal injection. The dose of guanosine was based on the in vivo use of nucleosides from cancer literature (13). Male C57 black mice weighing 15-25 g and Sprague-Dawley rats weighing 180-220 g (Harlan, Indianapolis, Indiana, USA) were anesthetized with intraperitoneal sodium pentobarbital $(50-70 \mathrm{mg} / \mathrm{kg})$ and placed on a homeothermic table to maintain core body temperature at $37^{\circ} \mathrm{C}$. Both renal pedicles were occluded via a midline incision for 32 minutes (14). Sham surgery consisted of an identical procedure with the exception of application of the microaneurysm clamps. Creatinine was determined by standard picric acid reaction in serum obtained from the tail vein or by cardiac puncture.

Light microscopy. Twenty-four hours after surgery, kidneys were perfusion fixed in situ with $4 \%$ paraformaldehyde. Kidneys were paraffin embedded, sectioned at 4 $\mu \mathrm{m}$, and stained using hematoxylin and eosin (H\&E). The percentage of tubules in the cortex and outer medulla that showed epithelial necrosis with luminal necrotic debris and tubular dilation were each quantified as follows: $0 \%,<10 \%, 10-25 \%, 26-75 \%$, and $>75 \%$. The estimates were performed by a blinded observer on coded sections (three to four sections per kidney and 10 to 12 fields per section) (15).

Fluorescence microscopy. A Zeiss confocal microscope (LSM 510; Carl Zeiss Inc., Thornwood, New York, USA), equipped with ultraviolet, argon, and helium lasers, was used. Pieces from the in situ fixed kidneys were preserved in $30 \%$ sucrose before $10-\mu \mathrm{m}$ frozen sections were obtained. Some sections were stained with FITC-phalloidin (for evaluation of actin cytoskeleton architecture and as a marker for proximal tubules) and 4',6-diamidine-2-phenylindole dihydrochloride (DAPI), a nuclear stain (Molecular Probes Inc., Eugene, Oregon, USA). Other sections were also costained with a rabbit polyclonal anti-human Tamm-Horsfall protein $\mathrm{Ab}$
(THP; Biogenesis Inc., Kingston, New Hampshire, USA) and a goat anti-rabbit Cy5-conjugated secondary $\mathrm{Ab}$ (Amersham Pharmacia Biotech, Piscataway, New Jersey, USA). This was used as a marker for Henle's loop.

Finally, separate sections were stained with TUNEL reagent (Promega Corp., Madison, Wisconsin, USA) and DAPI for in situ apoptosis detection. In brief, $10-\mu \mathrm{m}$ frozen sections were treated with $20 \mu \mathrm{g} / \mathrm{ml}$ proteinase $\mathrm{K}$ and then incubated in a nucleotide mixture containing fluorescein-12-dUTP and terminal transferase (TdT). Positive controls were pretreated with $1 \mathrm{U} / \mathrm{ml}$ DNase, and negative controls were incubated without TdT. TUNEL-positive nuclei were expressed as a percentage of total nuclei (DAPI positive) per field. Six to eight fields per section and two to three sections per kidney were examined in each experiment.

In some experiments, TUNEL-positive nuclei were expressed as a percentage of total nuclei of a particular tubular segment. This was done by labeling $5-\mu \mathrm{m}$ sections with TUNEL reagent and a directly contiguous $5-\mu \mathrm{m}$ section from the same tissue with phalloidin and anti-THP Ab. Overlay of the two sections allowed the localization of TUNEL-positive nuclei to various tubular segments.

Nucleotide extraction. Kidney tissue obtained 1 hour after sham surgery or ischemia with cutting forceps precooled in liquid nitrogen was crushed under liquid nitrogen and resuspended in $30 \%$ ice-cold acetonitrile and centrifuged at $16,000 \mathrm{~g}$ for 10 minutes at $-20^{\circ} \mathrm{C}$. The supernatant fraction, kept on ice, was gassed with $\mathrm{N}_{2}$ for 30 minutes to evaporate acetonitrile, and the nucleotides were assayed by HPLC as described in detail previously (12). The pellet's protein content was analyzed with Coomassie blue assay (Pierce Chemical Co., Rockford, Illinois, USA).

Plasma guanosine levels. Blood was collected into a chilled stopping solution: $26 \mu \mathrm{M}$ erythro-9-(2hydroxy-3-nonyl) adenine hydrochloride, $100 \mu \mathrm{M}$ dipyridamole, $4 \mathrm{mM}$ EDTA, $2 \mu \mathrm{g} / \mathrm{ml}$ indomethacin, and $100 \mathrm{U} / \mathrm{ml}$ heparin. The plasma was separated by centrifugation and the proteins precipitated with $10 \%$ trichloroacetic acid. The supernatant was washed with

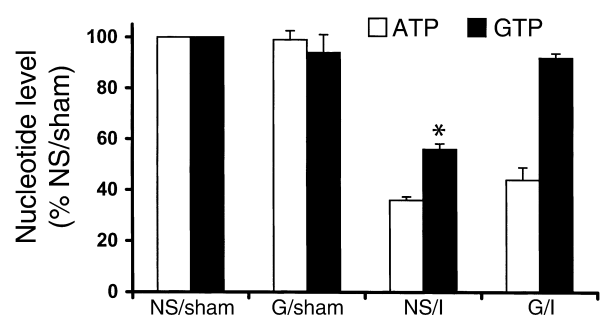

Figure 2

Effects of guanosine on renal nucleotide levels during reperfusion. Values are means \pm SE. Mice received normal saline (NS, $n=5)$ or guanosine (G, $n=5) 30 \mathrm{mg} / \mathrm{kg}$ intraperitoneally, 2 hours before 32-minute bilateral renal ischemia (I) or sham surgery. Renal tissues were obtained from each kidney 1 hour after surgery and processed for nucleotide determination ( ${ }^{*} P<0.02$, when GTP in NS/I was compared with GTP in G/I). 
tert-butyl-ether and the organic layer discarded. The sample was filtered through a cyano-bonded solidphase extraction cartridge and assayed by HPLC using established protocols (16).

Studies with LLC-PK1 cells. A4.8 clones of LLC-PK1 porcine proximal tubule cells were grown in $5 \% \mathrm{CO}_{2}$ at $37^{\circ} \mathrm{C}$ in DMEM with $10 \%$ FBS (Sigma Chemical Co.) and $5 \mathrm{mM}$ glucose (referred to as regular medium). For chemical anoxia, $0.1 \mu \mathrm{M}$ antimycin $\mathrm{A}$ was used in depleted media (DMEM without amino acids, glucose, or serum). Nucleotide extraction and measurements were done as described previously (12). Cell death was determined using a Zeiss confocal microscope. Both adherent and floating cells were visualized after staining with Hoechst 33342 and propidium iodide, as described previously (12). Cell viability was based on the criteria of trypan blue exclusion and cell adherence as described by others (10).

Statistics. Creatinine, guanosine, and nucleotide levels are expressed as means plus or minus SE. ANOVA was used to determine if differences among mean values reached statistical significance. Student $t$ test was used for comparisons between groups. Bonferonni adjustment was used for multiple comparisons. Fisher's exact test was used to determine if differences in histological parameters were significantly different.

\section{Results}

Effect of intraperitoneal guanosine on plasma guanosine levels. We first examined the time course of changes in plasma guanosine levels following an intraperitoneal guanosine administration in mice subjected to bilateral ischemia/reperfusion. As shown in Figure 1, there was a significant increase in plasma guanosine that peaked 2 hours after guanosine administration. Three to 7 hours after the intraperitoneal injection, levels were significantly lower than those at 2 hours but still slightly higher than baseline. Thus, guanosine was administered 2 hours before surgery in all subsequent experiments except when stated otherwise.

Effects of intraperitoneal guanosine on renal nucleotide levels during reperfusion. We have shown previously that renal GTP and ATP levels fall to less than $10 \%$ of control during renal artery clamp exceeding 20 minutes (12). After 6 to 8 hours of reperfusion, GTP and ATP normalized to preischemia levels. Furthermore, the decline in GTP levels during ischemia could not be prevented by guanosine supplementation (data not shown). Therefore, we investigated the effects of guanosine on GTP at an early time point during reperfusion. In the control kidneys, ATP was $18 \pm 3$ $\mathrm{pmol} / \mu \mathrm{g}$ protein and GTP was $6 \pm 2 \mathrm{pmol} / \mu \mathrm{g}$ protein. As shown in Figure 2, renal GTP levels were above $92 \%$ of control at 1 hour of reperfusion in the guanosine/ischemia mice (G/I) compared with $56 \%$ in normal saline/ischemia mice (NS/I). Furthermore, guanosine supplementation did not affect ATP levels significantly ( $44 \%$ in $\mathrm{G} / \mathrm{I}$ versus $36 \%$ in NS/I). Thus, guanosine selectively increased GTP levels to near baseline values after only 1 hour of reperfusion. Guanosine had no significant effects on GTP or ATP in the sham kidneys. Similar results were obtained in rats: in control sham kidneys, ATP was $28 \pm 4 \mathrm{pmol} / \mu \mathrm{g}$ protein and GTP $8 \pm 2 \mathrm{pmol} / \mu \mathrm{g}$ protein. Guanosine increased GTP after 1 hour reperfusion to $107 \%$ of control compared with $65 \%$ in the NS/I $(P<0.01)$. ATP levels were $49 \%$ in the G/I group versus $58 \%$ in the $\mathrm{NS} / \mathrm{I}(P=\mathrm{NS})$.

Effects of guanosine and enhanced GTP recovery on renal bistology at 24 hours. Histological sections of kidneys from NS-treated mice (NS/I group) removed 24 hours after ischemia showed extensive tubular necrosis, tubular dilation, and cast formation in the outer medulla (Figure 3). Sections from the guanosine-treated mice (G/I group) had similar histological features. Quantitation of these changes showed no significant differ-

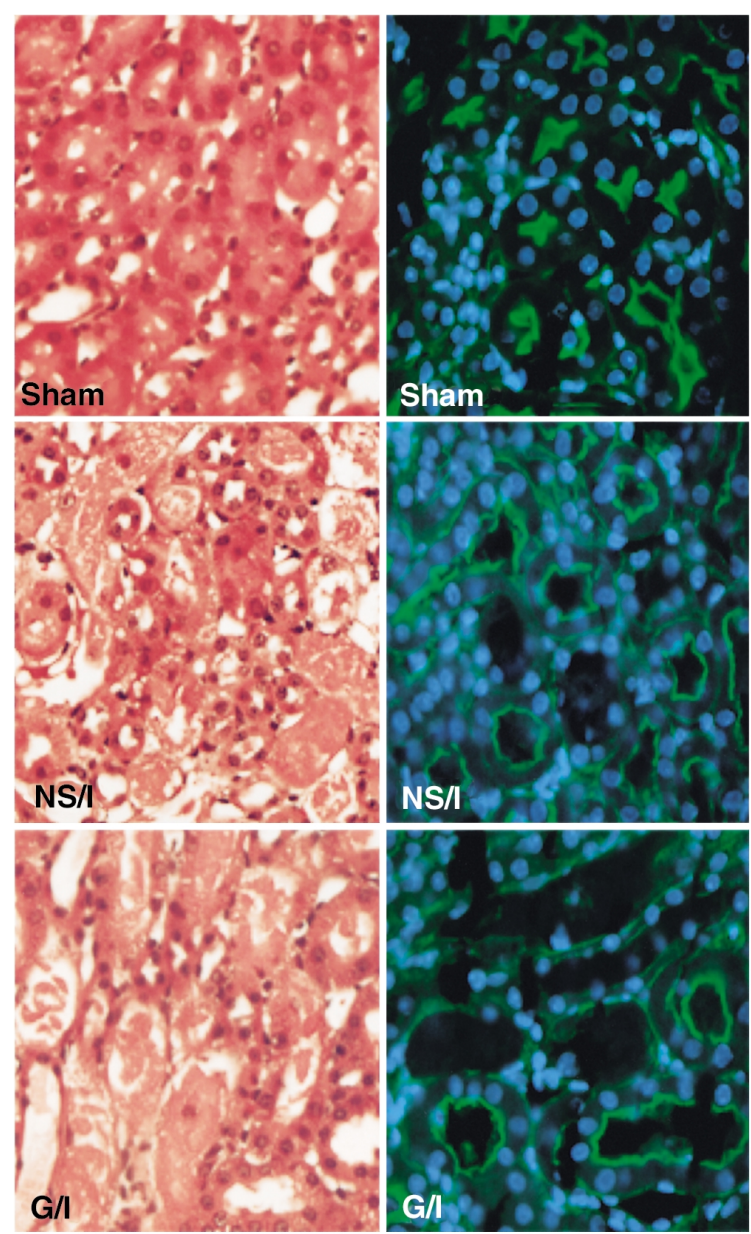

Figure 3

Effects of guanosine and enhanced GTP recovery on renal histology at 24 hours. Mice received normal saline (NS) or guanosine (G) 30 $\mathrm{mg} / \mathrm{kg}$ intraperitoneally 2 hours before renal ischemia (I) or sham surgery. Representative H\&E-stained sections from sham, NS/I, and G/I 24 hours after surgery are shown. Compared with the sham group, sections from both NS/I and G/I show patchy tubular dilatation, necrosis, and extensive cast formation. DAPI (blue) and FITCphalloidin (green) staining show significant loss and/or disruption of the apical actin rim in both NS/I and G/I as compared with sham. 
Table 1

Histological scoring of mouse kidney sections after ischemia and administration of guanosine or saline

\begin{tabular}{|c|c|c|c|c|c|c|c|c|}
\hline$\%$ tubules $^{\mathrm{A}}$ & & 0 & $<10 \%$ & $10-25 \%$ & $26-75 \%$ & $>75 \%$ & Total $^{\mathrm{B}}$ & $P$ value \\
\hline \multicolumn{9}{|l|}{ Cortex } \\
\hline Tubular necrosis & $\begin{array}{c}\text { NS } \\
\text { G }\end{array}$ & $\begin{array}{l}0 \\
0\end{array}$ & $\begin{array}{l}0 \\
0\end{array}$ & $\begin{array}{l}0 \\
0\end{array}$ & $\begin{array}{l}11 \\
16\end{array}$ & $\begin{array}{l}30 \\
30\end{array}$ & $\begin{array}{l}41 \\
46\end{array}$ & 0.16 \\
\hline Tubular dilatation & $\begin{array}{c}\text { NS } \\
\text { G }\end{array}$ & $\begin{array}{l}0 \\
0\end{array}$ & $\begin{array}{l}5 \\
8\end{array}$ & $\begin{array}{l}11 \\
16\end{array}$ & $\begin{array}{l}33 \\
22\end{array}$ & $\begin{array}{l}1 \\
0\end{array}$ & $\begin{array}{l}50 \\
46\end{array}$ & 0.15 \\
\hline Casts & $\begin{array}{c}\text { NS } \\
\text { G }\end{array}$ & $\begin{array}{l}22 \\
17\end{array}$ & $\begin{array}{l}4 \\
0\end{array}$ & $\begin{array}{l}10 \\
14\end{array}$ & $\begin{array}{l}14 \\
16\end{array}$ & $\begin{array}{l}0 \\
0\end{array}$ & $\begin{array}{l}50 \\
47\end{array}$ & 0.15 \\
\hline \multicolumn{9}{|l|}{ Medulla } \\
\hline Tubular necrosis & $\begin{array}{c}\text { NS } \\
\text { G }\end{array}$ & $\begin{array}{l}0 \\
0\end{array}$ & $\begin{array}{l}0 \\
0\end{array}$ & $\begin{array}{l}0 \\
0\end{array}$ & $\begin{array}{l}7 \\
5\end{array}$ & $\begin{array}{l}33 \\
41\end{array}$ & $\begin{array}{l}40 \\
46\end{array}$ & 0.38 \\
\hline Tubular dilatation & $\begin{array}{c}\text { NS } \\
\text { G }\end{array}$ & $\begin{array}{l}0 \\
1\end{array}$ & $\begin{array}{l}0 \\
3\end{array}$ & $\begin{array}{l}19 \\
24\end{array}$ & $\begin{array}{l}21 \\
18\end{array}$ & $\begin{array}{l}0 \\
0\end{array}$ & $\begin{array}{l}40 \\
46\end{array}$ & 0.22 \\
\hline Casts & $\begin{array}{c}\text { NS } \\
\text { G }\end{array}$ & $\begin{array}{c}8 \\
15\end{array}$ & $\begin{array}{l}4 \\
0\end{array}$ & $\begin{array}{l}12 \\
16\end{array}$ & $\begin{array}{l}16 \\
14\end{array}$ & $\begin{array}{l}0 \\
0\end{array}$ & $\begin{array}{l}40 \\
45\end{array}$ & 0.16 \\
\hline
\end{tabular}

AThe percentage of tubules in each field with tubular necrosis, or dilatation, or containing casts were quantified as in Methods. ${ }^{B}$ Numbers represent number of fields with the given histological score. apoptosis in renal tissues. As shown in Figure 4, kidneys from mice in the NS/I group showed extensive TUNEL-positive staining (Figure 4c), predominantly in the outer medulla. In contrast, kidneys from mice in the G/I group had very little TUNELpositive staining (Figure 4d). Furthermore, because the TUNEL stain can occasionally be nonspecific, we examined nuclear morphology with DAPI staining at higher magnification $(\times 60)$. In Figure $4 e$, nuclei from the NS/I group showed heavy staining and condensation of chromatin. The inset in Figure 4e shows $\times 120$ magnification of a single nucleus. Fragmentation of chromatin into several smaller bodies is seen, a hallmark of apoptosis. Figure $4 \mathrm{f}$ is a $\times 60$ magnification of nuclei from $G / I$

ences between these groups (Table 1). In addition, staining with phalloidin demonstrated comparable disruption and thinning of apical actin rim between the two groups. The sham surgery group showed a wellformed, thick, and continuous actin rim (Figure 3). Thus, guanosine and enhanced GTP recovery after ischemia did not have a significant effect on the morphologic consequences of renal ischemia in this mouse model. The histology and actin architecture of the sham group that received guanosine were normal and indistinguishable from the NS-treated sham group.

Similar changes were seen in rat kidneys. The majority of sections of outer medulla from both the NS/I and $\mathrm{G} / \mathrm{I}$ rats showed evidence of tubular necrosis in $>75 \%$ of tubules examined. The apical actin rim also showed comparable thinning and disruption in both groups (data not shown).

Effects of guanosine and enhanced GTP recovery on renal cell apoptosis at 24 hours. At 24 hours after ischemia, guanosine had a remarkable effect on the occurrence of

\section{Figure 4}

Effects of guanosine and enhanced GTP recovery on renal cell apoptosis at 24 hours. Mice were treated as detailed in legend of Figure 2 . Kidney sections were obtained at 24 hours and costained with TUNEL and DAPI. (a) A negative control (NC; TdT omitted) and (b) a DNase-treated positive control (PC) for TUNEL. All TUNEL-positive nuclei had bright green fluorescence. (c) A representative field showing the medulla from NS/I group. Extensive TUNEL-positive staining is seen and is localized primarily to the tubular epithelium (dark green autofluorescence). (d) A representative field from the medulla of the G/I group showing lack of TUNEL-positive staining. (e) A magnification of TUNEL-positive nuclei from NS/I mice, with only the DAPI channel turned on. $\times 60$. Nuclei are condensed, dysmorphic, and show heavy staining. Inset shows a single nucleus. $\times 120$. Typical apoptotic fragmentation of chromatin into four small bodies is seen. (f) A magnification of TUNEL-negative nuclei from G/I mice. $\times 60$. They show lack of condensation and faint DAPI staining. mice. They do not show condensation and stain very faintly with DAPI.

In addition, we confirmed the presence of apoptotic nuclei in the NS/I group by high-magnification $(\times 100)$ light microscopy of H\&E-stained tissues. As shown in Figure 5, a and b, very few if any apoptotic nuclei were observed in the G/I group. In contrast, multiple apoptotic nuclei with classic condensation and fragmentation can be seen in the NS/I group (Figure 5, c-f). Elec-
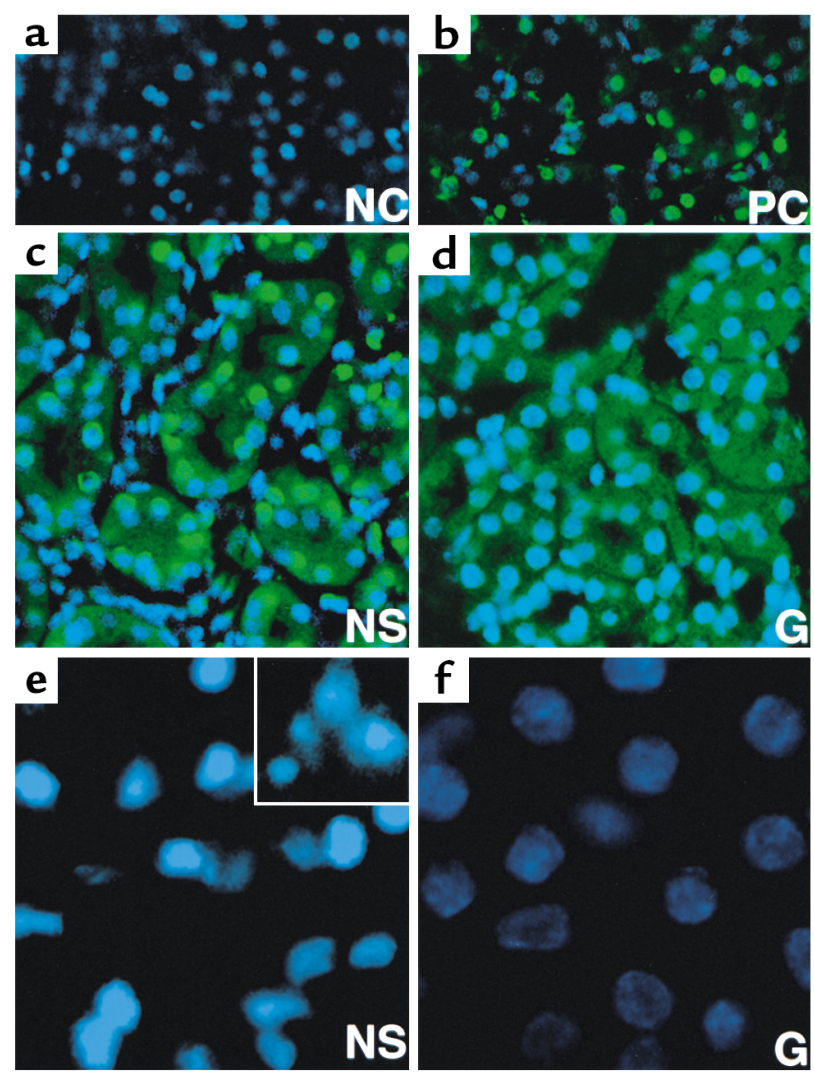


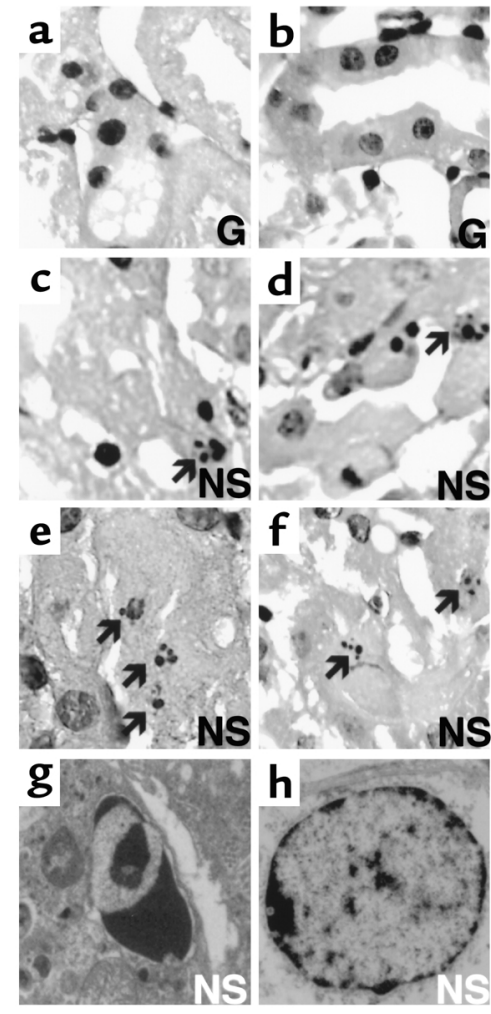

\section{Figure 5}

Morphologic characterization of apoptosis by light and electron microscopy. Mice received normal saline (NS) or guanosine (G) 30 $\mathrm{mg} / \mathrm{kg}$ intraperitoneally 2 hours before renal ischemia or sham surgery. Representative H\&E-stained sections 24 hours after ischemia from the guanosine-treated group are shown ( $\mathbf{a}$ and $\mathbf{b}$ ). Nuclei with predominantly necrotic features are observed. They have degraded chromatin without formation of discrete, membrane-bound fragments. (c, d, e, and f) From the NS-treated group. Classic apoptotic bodies with dense condensation and fragmentation of chromatin into discrete fragments are seen (arrows). ( $\mathbf{g}$ and $\mathbf{h}$ ) Electron microscopic photomicrographs of sections from the normal saline group show clear apoptotic (g) and necrotic (h) morphologies.

tron microscopic examination of tissues from the NS/I group clearly identified two types of nuclei in addition to normal ones: apoptotic nuclei (Figure $5 \mathrm{~g}$ ) and necrotic ones (Figure $5 \mathrm{~h}$ ). Thus, the apoptosis found by TUNEL was confirmed by fluorescence, light, and electron microscopic criteria.

The distribution of TUNEL-positive staining between outer medulla and cortex in the four groups is shown in Figure 6 . The highest TUNEL-positive staining was in the medulla of the NS/I group and represented $28 \%$ of all nuclei stained in this region. In the G/I group, the number of TUNEL-positive nuclei was reduced significantly to $3 \%$. There was less TUNEL positivity in the cortex of the NS/I group (8\%), but this was still reduced in the $\mathrm{G} / \mathrm{I}$ group to less than $2 \%$. Similar results were observed in rats. The NS/I group showed 33\% TUNELpositive nuclei in the medulla. This was reduced in the $\mathrm{G} / \mathrm{I}$ group to less than $5 \%$. There was minimal cortical TUNEL positivity $(<2 \%)$ in all groups.
Distribution of TUNEL-positive nuclei among various medullary structures. We next examined the distribution of TUNEL-positive nuclei in the NS/I group among various medullary segments of the nephron. As detailed in Methods, this was done by overlay of TUNEL-stained sections with directly contiguous sections stained with phalloidin and anti-THP Ab (17). As shown in Figure 7, TUNEL-positive nuclei (Figure 7a) and proximal tubules, distal tubules, as well as loop segments (Figure 7b), could easily be overlaid to yield a composite picture (Figure 7c) showing the TUNEL distribution among various segments.

Figure $7 \mathrm{~d}$ shows a quantitative estimate of the TUNEL distribution obtained by counting ten fields similar to that shown in Figure 7c. On average, there was no difference between proximal, distal, and loop $\left(\mathrm{THP}^{+}\right)$segments, with each having $26-29 \%$ of their nuclei TUNEL positive. Of note is the presence of about 44\% TUNEL-positive nuclei in tubular lumens (normalized to all luminal nuclei). Few TUNEL-positive nuclei were noted in interstitial and vascular structures.

Effects of guanosine and enhanced renal GTP levels on renal function at 24 hours. The results presented above show that guanosine and increased GTP levels did not alter the histological findings at 24 hours but did reduce significantly medullary apoptosis. To test if this reduction in apoptosis had any impact on function, we measured serum creatinine in all groups at 24 hours. As shown in Figure 8, the NS/I group had a mean creatinine of $2.1 \pm 0.3 \mathrm{mg} / \mathrm{dl}$. This was significantly reduced to $0.76 \pm 0.12 \mathrm{mg} / \mathrm{dl}$ in the G/I group. In fact, the creatinine in the G/I group was comparable to that of the two sham groups. Guanosine and enhanced GTP levels had a similar protective effect in the rat: at 24 hours, creatinine was $3.2 \pm 0.3 \mathrm{mg} / \mathrm{dl}$ in the NS/I group compared with $1.4 \pm 0.2 \mathrm{mg} / \mathrm{dl}$ in the G/I group $(n=3, P<0.01)$.

We further examined the window of efficacy of guanosine. We found that guanosine was most protec-

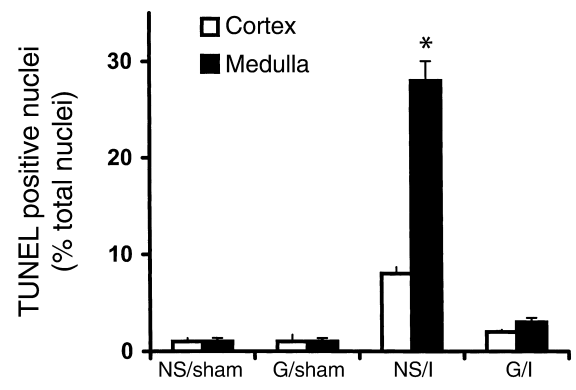

\section{Figure 6}

Quantitative evaluation ofTUNEL-positive nuclei in cortex and medulla. Values are means \pm SE and represent number of TUNEL-positive nuclei expressed as percentage of total number of nuclei. Mice were treated as detailed in the legend of Figure 2. Kidney sections were obtained at 24 hours and processed for TUNEL and DAPI staining as detailed in Methods. Number of fields counted: 20 for G/I, 35 for $\mathrm{NS} / \mathrm{I}, 8$ for $\mathrm{G} /$ sham, and 8 for NS/sham. ( $P<0.01$ when medullary TUNEL-positive nuclei in NS/I were compared to all other groups). 

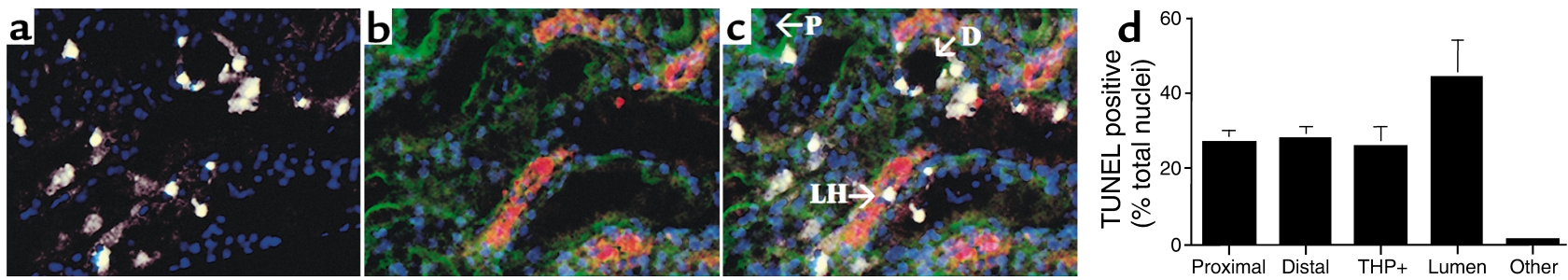

\section{Figure 7}

Distribution ofTUNEL-positive nuclei among various nephronal segments. (a) A 5- $\mu$ m thick section from NS/I mice stained with TUNEL and DAPI. The TUNEL positive nuclei were pseudocolored white instead of green to allow clear distinction from the green FITC-phalloidin. (b) A $5-\mu \mathrm{m}$ section immediately contiguous to the one shown in $\mathbf{a}$ is stained with the actin marker FITC-phalloidin (bright green) to identify proximal tubules (P). Distal tubules (D) show minimal or no staining. The section was costained with anti-THP Ab and a Cy5-conjugated second$\operatorname{ary} \mathrm{Ab}(\mathrm{red})$. This is predominantly a marker for Henle's loop segments (LH). (c) An overlay of $\mathbf{a}$ and $\mathbf{b}$ allows the localization of TUNEL-positive nuclei to various tubular segments. (d) A quantitative estimate of the distribution of TUNEL-positive nuclei normalized to total number of DAPI-positive nuclei for each particular tubular segment or lumen. Other refers to interstitial or vascular TUNEL-positive nuclei.

tive when given 2 hours before ischemia. Mean creatinine was no different than in the control postischemic group when guanosine was given 4 or 8 hours before ischemia or 4 or 8 hours after ischemia. When guanosine was given at the time of ischemia or 2 hours after ischemia, the improvement in mean creatinine did not reach statistical significance. Peak guanosine levels occur 2 to 3 hours after an intraperitoneal injection (Figure 1), coinciding well with the enhanced GTP levels observed 1 hour after ischemia ( 3 hours after guanosine administration).

Effects of guanosine on nucleotide content, cell viability, and apoptosis after chemical anoxia in LLC-PK1 cells. Because of the cellular complexity of kidney tissues, we investigated if the reported changes in nucleotides and cell death can be reproduced in tubular cells in culture. For this purpose, we used the well-characterized LLC-PK1 proximal tubular cell line to model the effects of guanosine. Resting ATP and GTP levels were $36 \pm 4 \mathrm{pmol} / \mu \mathrm{g}$ and $8 \pm 2$ $\mathrm{pmol} / \mathrm{\mu g}$ protein, respectively $(n=20)$. The addition of up to $200 \mu \mathrm{M}$ guanosine for 2 hours increased ATP to 40 $\pm 3 \mathrm{pmol} / \mu \mathrm{g}$ and GTP to $9 \pm 2 \mathrm{pmol} / \mu \mathrm{g}$ protein $(n=5)$. Both changes were not statistically significant.

Next we investigated the effects of guanosine on nucleotide recovery after antimycin A-induced chemical anoxia. As shown in Figure 9a, 45 minutes' exposure to $0.1 \mu \mathrm{M}$ antimycin A in depleted media reduced ATP to $5 \% \pm 2 \%$ and GTP to $8 \% \pm 2 \%$ of control levels. After 2 hours' recovery in regular media, ATP was $33 \% \pm 3 \%$ and GTP was $49 \% \pm 4 \%$ of control values. When $200 \mu \mathrm{M}$ guanosine was included in the recovery medium for 2 hours, ATP was $35 \% \pm 2 \%$ and GTP was $88 \% \pm 5 \%$ of control (Figure 9b). Thus, guanosine enhanced early selective GTP recovery after chemical anoxia.

We then quantitated cell viability after chemical anoxia and recovery for 24 hours. The presence of guanosine significantly increased cell viability at 24 hours: viability was $70 \% \pm 8 \%$ in the guanosine-treated group as compared with $44 \% \pm 6 \%$ in the control group $(P<0.02)$. As shown in Figure 9, $\mathrm{c}$ and $\mathrm{d}$, the increase in viability in the guanosine group primarily was due to a reduction in apoptotic cell death. Indeed, the guanosine-treated group showed less than $10 \%$ apoptosis at 8,12 , and 24 hours as compared with $40 \%$ in the control group (the representative fields shown in Figure 9 are from a 24-hour time point). Neither group showed any significant necrosis at these three time points as evidenced by exclusion of propidium iodide. Thus, guanosine supplementation reduces apoptosis and increases viability after in vitro chemical anoxia in LLCPK1 proximal tubular cells.

\section{Discussion}

In this article, we extend our findings relating apoptosis and GTP depletion in cell culture to the in vivo ischemia setting. Indeed, our results in both mice and rats show that guanosine selectively repletes renal GTP levels after 1 hour of reperfusion. This early increase in GTP is accompanied by a significant reduction in medullary apoptosis and a remarkable protective effect on GFR. Therefore, these results introduce two important concepts to be discussed. First is the relationship of GTP levels to apoptosis and second is the relationship of apoptosis to renal function.

The depletion of guanine nucleotides has been implicated in the induction of apoptosis in anoxic cells in

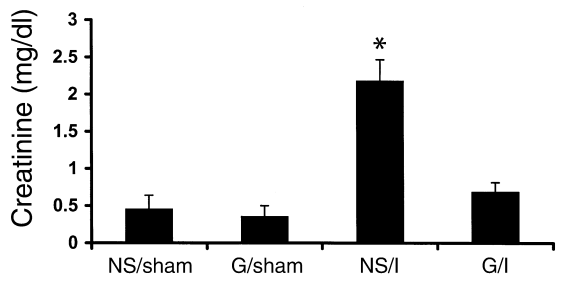

\section{Figure 8}

Effects of guanosine and enhanced renal GTP levels on renal function at 24 hours. Values are means \pm SE. Creatinine levels at 24 hours are shown after administration of normal saline (NS) or guanosine (G) 2 hours before sham surgery or bilateral renal ischemia. Mean creatinine was significantly higher in the NS/I mice than in the other groups. ( ${ }^{*} P<0.01, n=6$ for NS/I and G/I groups). 

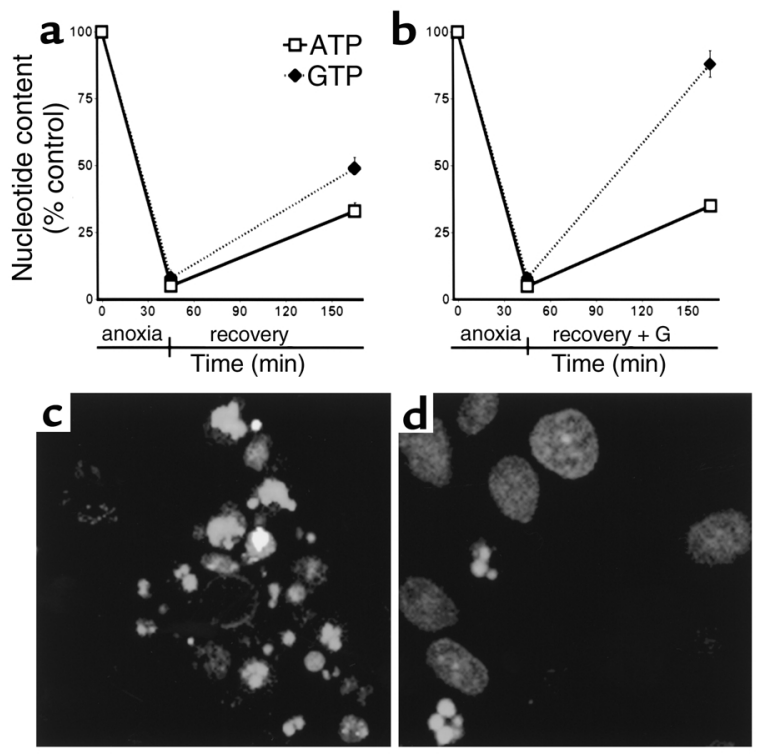

\section{Figure 9}

Effects of guanosine on nucleotide levels and apoptosis after chemical anoxia recovery in LLC-PK1 cells. Values are means \pm SE. (a) LLC-PK1 cells were treated for 45 minutes with $0.1 \mu \mathrm{M}$ antimycin $\mathrm{A}$ in depleted media followed by recovery for 2 hours. Nucleotides were measured at 45 minutes of depletion $(n=7)$ and at 2 hours of recovery $(n=6)$. (b) Cells were treated identically except for the addition of $200 \mu \mathrm{M}$ guanosine to the recovery medium $(n=6)$. ( $c$ and $\mathbf{d}$ ) Representative fields of confocal microscopic images of LLC-PK1 cells at 24 hours after recovery from chemical anoxia. Cells were costained with Hoechst 33342 and propidium iodide as detailed in Methods. In c, cells recovered in regular media. Apoptotic features such as condensation and fragmentation of chromatin is seen in most cells in the field. In d, cells recovered in the presence of $200 \mu \mathrm{M}$ guanosine. Most nuclei showed normal morphology, and only few had apoptotic features.

culture (12) and in settings other than anoxia or ischemia $(18,19)$. The exact mechanism is unknown, but two hypotheses have been proposed: first is the small size of the GTP pools compared with ATP pools $(20,21)$. In most cells, the GTP/ATP ratio is around $1: 5$, and thus even minor reductions in GTP could be rate limiting for nucleic acid synthesis and hence trigger apoptosis. The second mechanism proposes that GTP depletion could alter the activation states of GTPases of the Ras superfamily $(18,22)$. Most members of this family are oncogenes and their inactivation has been linked to apoptosis (23).

Our results in mice and rats are in remarkable agreement with our in vitro findings and strongly implicate GTP depletion in renal tubular apoptosis. However, the complexity of in vivo renal ischemia imposes significant caution to the proposal of a direct link between GTP and apoptosis. For example, the reported GTP levels are average numbers from both cortex and medulla. Thus, the exact GTP changes in these two regions could not be dissected further. In addition, the presence of multiple cell types in these regions also imposes some restriction on our results. However, tubular cells are far more abundant than other cell types, and this justifies our assumption that the reported levels predominantly represent tubular GTP. Furthermore, we were able to reproduce the changes in GTP (and apoptosis) in LLC-PK1 proximal tubular cells in culture. Thus, the reported changes in nucleotides in renal tissues most likely reflect events in tubular cells. Improvements in the sampling technique for GTP measurements will allow a better evaluation of the role of this nucleotide in cortical versus medullary regions.

Recent studies have reported a protective effect of guanosine against neuronal hypoxic injury in culture $(24,25)$. This beneficial effect was ascribed to an enhancement in ATP production. Our in vivo and in vitro studies do not support such a role for guanosine in renal tubular cells. After guanosine administration, only GTP levels were increased with minimal or no effect on ATP levels. Selective effects of guanosine on GTP as opposed to a nonselective increase in all nucleotides could be related to the doses of guanosine used or cellspecific differences in purine metabolic pathways.

Another degree of caution is imposed by our limited knowledge of possible direct effects of guanosine unrelated to nucleotide production. These could include hemodynamic, vascular, or anti-inflammatory properties. However, unlike adenosine, guanosine has no known receptors or second messenger cascades. It is taken up by specific transporters into cells and then proceeds via the salvage pathway to generate guanine nucleotides (26). This pathway is catalyzed by the ratelimiting enzyme hypoxanthine phosphoribosyl transferase (HPRT). Studies in HPRT-null mice will uncover possible direct effects of guanosine. These mice cannot synthesize GTP from guanosine and therefore will be useful to probe for direct effects of guanosine (27). Nevertheless, the histological findings in the $\mathrm{G} / \mathrm{I}$ group revealed morphological damage similar to that seen in the NS/I group. These data and those in LLC-PK1 cells argue against significant beneficial hemodynamic or anti-inflammatory properties of guanosine. They also underscore the difference in apoptosis as the main underlying mechanism for the improved function.

Our confidence in the TUNEL assay as a reporter of apoptosis relies on two facts. First is the confirmation of apoptosis by morphological criteria. Indeed, condensation and fragmentation of nuclear chromatin remains the gold standard for the diagnosis of apoptosis. Second, the lack of TUNEL positivity in the G/I group (despite extensive necrosis comparable to that seen in NS/I group) argues against nonspecific or necrotic readings of the TUNEL assay (28). Thus, both the TUNEL assay and nuclear morphology suggest that guanosine significantly reduced medullary apoptosis after ischemia-reperfusion injury.

Apoptosis has been reported after ischemia and its inhibition often leads to improved function in many organ systems (29-33). The pathophysiology of this phenomenon is more easily understood in settings such as stroke or myocardial infarction. This relates to preservation of neuronal and cardiac tissue mass, which in 
turn affects function directly. In the kidneys, the link between inhibition of apoptosis and the improved GFR is less obvious. A recent study proposed that apoptosis is proinflammatory and thus detrimental to function (30). Our histological findings do not support such an interpretation. In fact, a lack of correlation between histological evidence of injury and renal function has been found in both human acute renal failure $(34,35)$ and in animal models of renal injury (36). It is possible that prevention of apoptotic death in nearly $30 \%$ of medullary tubular cells confers a protective effect on overall tubular integrity and nephron function.

In conclusion, we report a novel finding regarding the protective effect of guanosine against renal ischemic injury in vivo. Our results show that guanosine selectively increases GTP and has no significant effects on ATP pools. Thus, this protective effect is likely secondary to enhanced GTP levels leading to reduced medullary programmed cell death. Our study highlights the importance of cellular GTP levels as targets for therapeutic intervention. It also proposes guanosine as a potential tool to manipulate GTP levels in vivo and alter the outcome of ischemic injury to the kidneys.

\section{Acknowledgments}

This work was supported in part by the Showalter Award (to P.C. Dagher), NIH grant DK-02364 (to K.J. Kelly), and by start-up funds from Indiana University (to P.C. Dagher and K.J. Kelly). The authors are grateful to Bruce Molitoris for support throughout this project, to Serafina Salamo for technical assistance, and Janice Pennington for help with electron microscopy.

1. Kelly, K.J., and Molitoris, B.A. 2000. Acute renal failure in the new millennium: time to consider combination therapy. Semin. Nephrol. 20:4-19. 2. Star, R. Treatment of acute renal failure. 1998. Kidney Int. 54:1817-1831.

3. Dubose, T.D., Jr., et al. 1997. Acute renal failure in the 21 st century: recommendations for management and outcomes assessment. Am. J. Kidney Dis. 29:793-799.

4. Safirstein, R. 2001. Endothelin: the yin and yang of ischemic acute renal failure. Kidney Int. 59:1590-1591.

5. Goligorsky, M.S. 2000. Endothelial cell dysfunction and nitric oxide synthase. Kidney Int. 58:1360-1376.

6. Molitoris, B., and Marrs, J. 1999. The role of cell adhesion molecules in ischemic acute renal failure. Am. J. Med. 106:583-592.

7. Hammerman, M.R., and Miller, S.B. 1997. Effects of growth hormone and insulin-like growth factor I on renal growth and function. J. Pediatrics. 131(Suppl.):S17-S19.

8. Thadani, R., Pascual, M., and Bonventre, J.V. 1996. Acute renal failure. N. Engl. J. Med. 334:1448-1460.

9. Lieberthal, W., Koh, J.S., and Levine, J.S. 1998. Necrosis and apoptosis in acute renal failure. Semin. Nephrol. 18:505-518.

10. Lieberthal, W., Menza, S.A., and Levine, J.S. 1998. Graded ATP depletion can cause necrosis or apoptosis of cultured mouse proximal tubular cells. Am. J. Physiol. 274:F315-F327.

11. Doctor, R.B., Bacallao, R., and Mandel, L.J. 1994. Method for recovering ATP content and mitochondrial function after chemical anoxia in renal cell cultures. Am. J. Physiol. 266:C1803-C1811.

12. Dagher, P.C. 2000. Modeling ischemia in vitro: selective depletion of adenine and guanine nucleotide pools. Am. J. Physiol. 279:C1270-C1277.

13. Kim, S.G., et al. 1997. Enhanced anti-tumour effects of acriflavine in combination with guanosine in mice. J. Pharm. Pharmacol. 49:216-222.

14. Kelly, K.J., et al. 1996. Intercellular adhesion molecule-1 deficient mice are protected against ischemic renal injury. J. Clin. Invest. 97:1056-1063.

15. Kelly, K.J., Williams, W.W., Colvin, R.B., and Bonventre, J.V. 1994. Antibody to intercellular adhesion molecule- 1 protects the kidney against ischemic injury. Proc. Natl. Acad. Sci. USA. 91:812-816.

16. Feng, J.D., and Yeung, P.K. 2000. A simple high-performance liquid chromatography assay for simultaneous measurement of adenosine, guanosine, and the oxypurine metabolites in plasma. Ther. Drug Monit. 22:177-183.

17. Balster, D.A., O’Dorisio, M.S., Summers, M.A., and Turman, M.A. 2001. Segmental expression of somatostatin receptor subtypes sst(1) and sst(2) in tubules and glomeruli of human kidney. Am. J. Physiol. 280:F457-F465.

18. Li, G., et al. 1998. Prolonged depletion of guanosine triphosphate induces death of insulin-secreting cells by apoptosis. Endocrinology. 139:3752-3762.

19. Vitale, M., et al. 1997. IMP dehydrogenase inhibitor, tiazofurin, induces apoptosis in K562 human erythroleukemia cells. Cytometry. 30:61-66.

20. Linke, S.P., Clarkin, K.C., Di Leonardo, A., Tsou, A., and Wahl, G.M. 1996. A reversible, p53-dependent G0/G1 cell cycle arrest induced by ribonucleotide depletion in the absence of detectable DNA damage. Genes Dev. 10:934-947.

21. Liu, Y., Bohn, S.A., and Sherley, J.L. 1998. Inosine-5'-monophosphate dehydrogenase is a rate-determining factor for p53-dependent growth regulation. Mol. Biol. Cell. 9:15-28.

22. Hata, Y., Natsumeda, Y., and Weber, G. 1993. Tiazofurin decreases RasGTP complex in K562 cells. Oncol. Res. 5:161-164.

23. Chen, C.Y., Liou, J., Forman, L.W., and Faller, D.V. 1998. Differential regulation of discrete apoptotic pathways by Ras. J. Biol. Chem. 273:16700-16709.

24. Jurkowitz, M.S., Litsky, M.L., Browning, M.J., and Hohl, C.M. 1998. Adenosine, inosine, and guanosine protect glial cells during glucose deprivation and mitochondrial inhibition: correlation between protection and ATP preservation. J. Neurochem. 71:535-548.

25. Litsky, M.L., Hohl, C.M., Lucas, J.H., and Jurkowitz, M.S. 1999. Inosine and guanosine preserve neuronal and glial cell viability in mouse spinal cord cultures during chemical hypoxia. Brain Res. 821:426-432.

26. Flanagan, S.A., and Meckling-Gill, K.A. 1997. Characterization of a novel $\mathrm{Na}+$-dependent, guanosine-specific, nitrobenzylthioinosine-sensitive transporter in acute promyelocytic leukemia cells. J. Biol. Chem. 272:18026-18032.

27. Hooper, M., Hardy, K., Handyside, A., Hunter, S., and Monk, M. 1987. HPRT-deficient (Lesch-Nyhan) mouse embryos derived from germline colonization by cultured cells. Nature. 326:292-295.

28. Andrade, L., Vieira, J.M., and Safirstein, R. 2000. How cells die counts. Am. J. Kidney Dis. 36:662-668.

29. Cheng, Y., et al. 1998. Caspase inhibitor affords neuroprotection with delayed administration in a rat model of neonatal hypoxic-ischemic brain injury. J. Clin. Invest. 101:1992-1999.

30. Daemen, M.A., et al. 1999. Inhibition of apoptosis induced by ischemiareperfusion prevents inflammation. J. Clin. Invest. 104:541-549.

31. Schierle, G.S., et al. 1999. Caspase inhibition reduces apoptosis and increases survival of nigral transplants. Nat. Med. 5:97-100.

32. Yaoita, H., Ogawa, K., Maehara, K., and Maruyama, Y. 1998. Attenuation of ischemia/reperfusion injury in rats by a caspase inhibitor. Circulation. 97:276-281.

33. Beeri, R., et al. 1995. Rapid DNA fragmentation from hypoxia along the thick ascending limb of rat kidneys. Kidney Int. 47:1806-1810.

34. Racusen, L.C. 1997. Pathology of acute renal failure: structure/function correlations. Adv. Ren. Replace. Ther. 42:3-16.

35. Solez, K., Morel-Maroger, L., and Sraer, J.D. 1979. The morphology of "acute tubular necrosis" in man; analysis of 57 renal biopsies and a comparison with the glycerol model. Medicine. 58:362-376.

36. Sabbatini, M., et al. 1994. Functional versus structural changes in the pathophysiology of acute ischemic renal failure in aging rats. Kidney Int. 45:1355-1361. 